تجزيه زنتيكى صفات زراعى در نسلهاى حاصل از تلاقى لاينهاى اينبرد



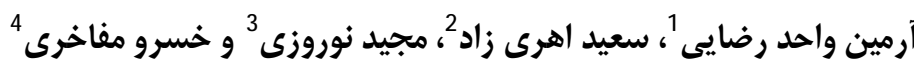

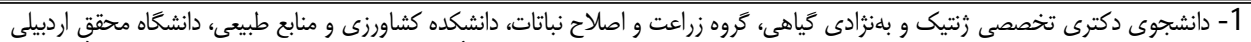

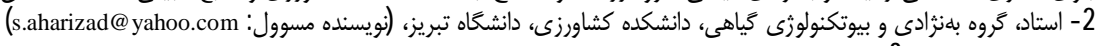

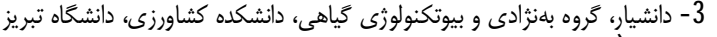



تاريخ دريافت:1398/2/16

صفحه: 76 تا 85

קצيده

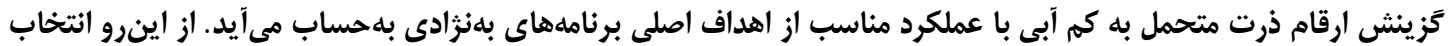

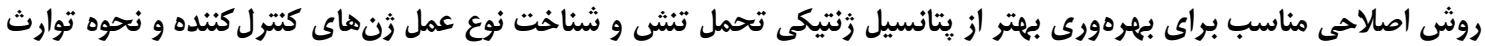

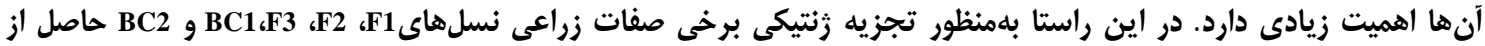

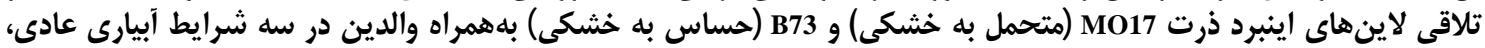

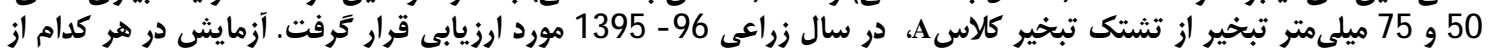

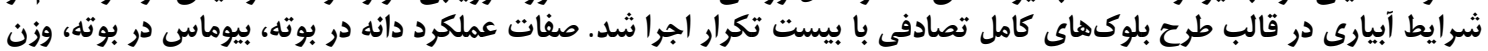

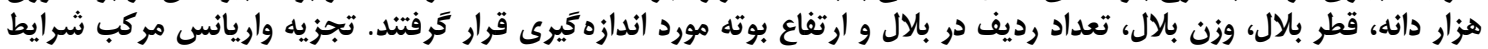

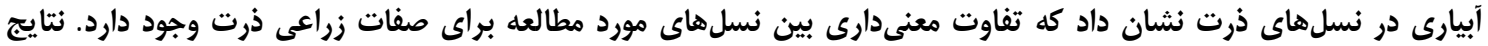

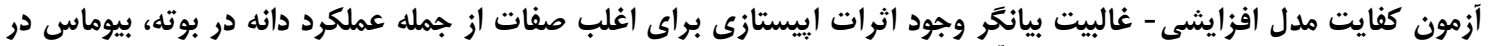

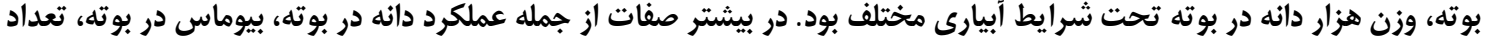

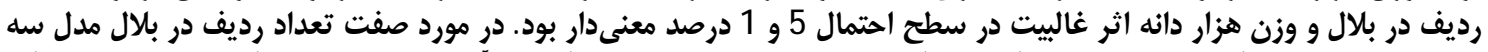

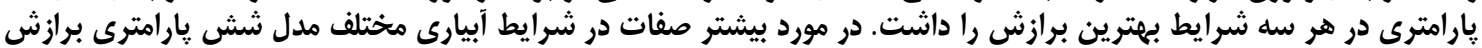

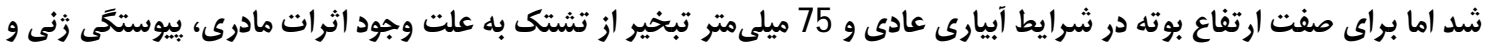

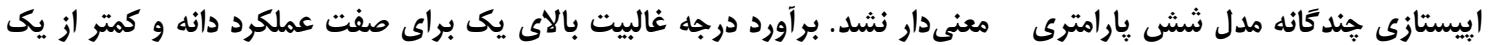

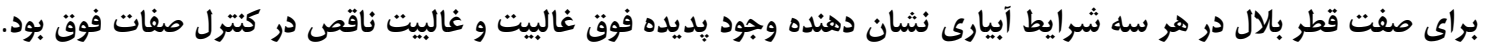



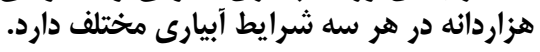

وازههاى كليدى: اثرات زنى، تجزيه ميانكين نسلها، وراثت پذيرى، غالبيت، ذرت

گياه در معرض تنش بستخى دارد و در نتيجه تنش كمبود آب،

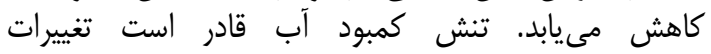

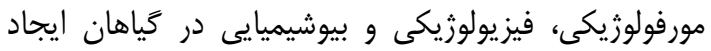

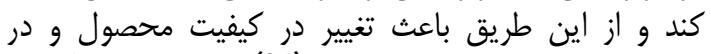
نهايت كاهش عملكرد كياه شود (31). تنش كمبر كمبود آب آب از

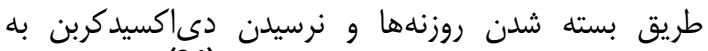
كلرويلاست باعث كاهش فتوسنتز مى شود (21). به بيان ديخ ديخر تنش كمآبى باعث كاهش هدايت روزنهاي واعث و فتوسنتز

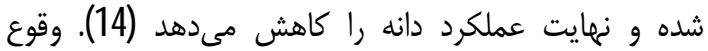

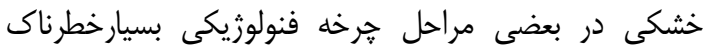

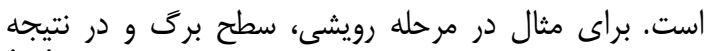

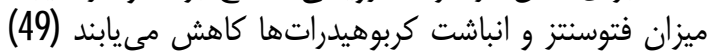

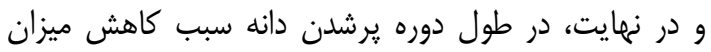

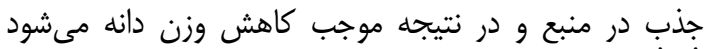

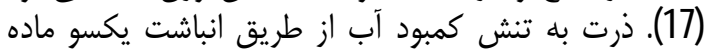

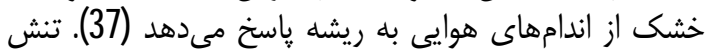

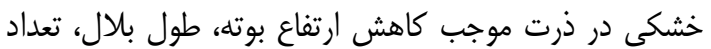

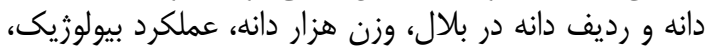

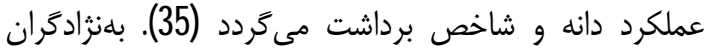
سالها است كه براى توليد واريته هايى با كيفيت بهترد، متحمل دمل
مقدمه (Zea mays L.) يكى از مهمترين

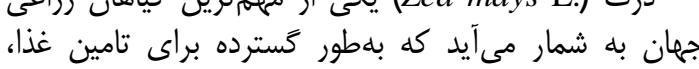

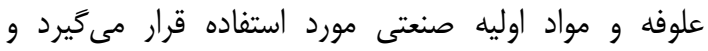

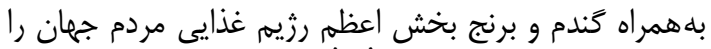

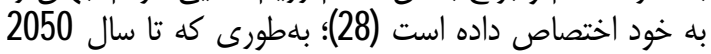

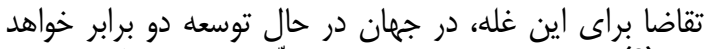

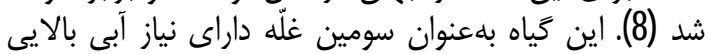

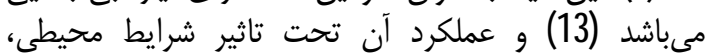

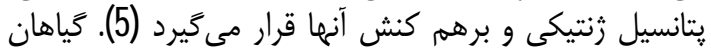

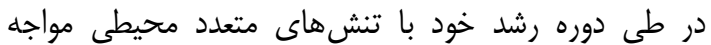

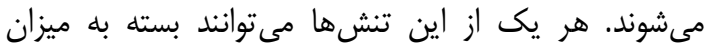

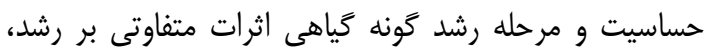

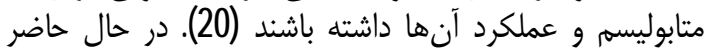

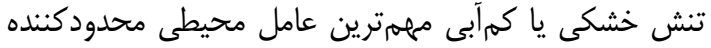

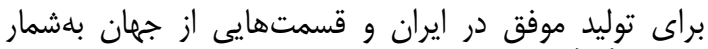

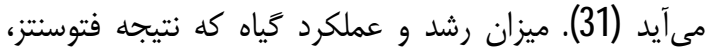

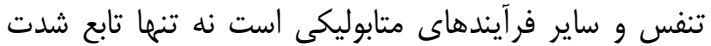

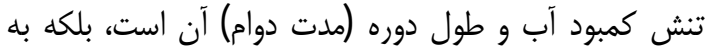

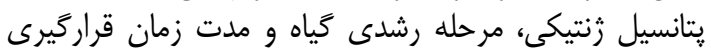


ثنهاى كنترل كننده و وراثت تعدادى از صفات زراعى در

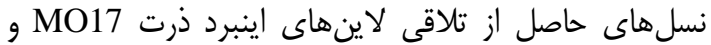
B73 در شرايط تنش كماصل تأبى بود.

\section{مواد و روشها}

اين أزمايش در سال 1396 در مزرعه تحقيقاتى دانشكده



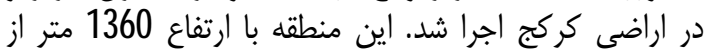

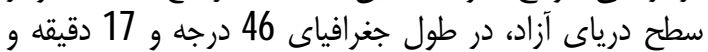

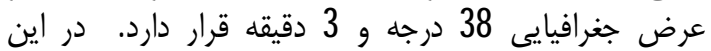

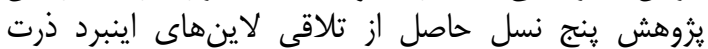

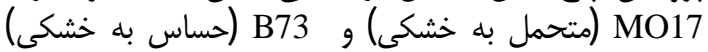

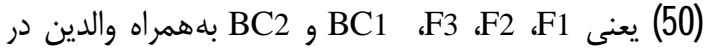

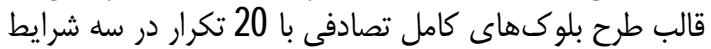

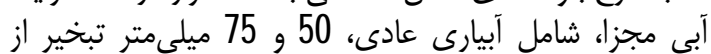

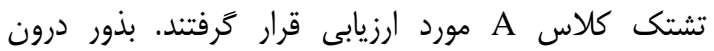

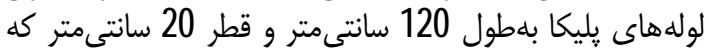

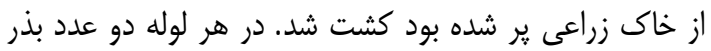

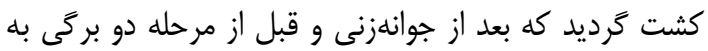

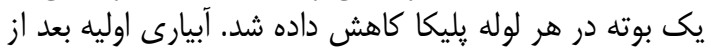

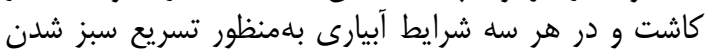



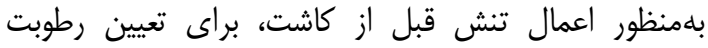

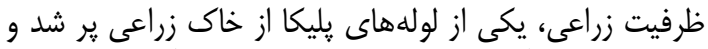

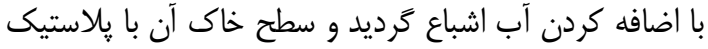

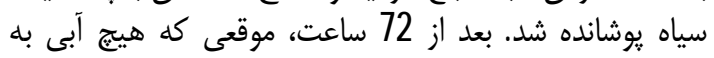

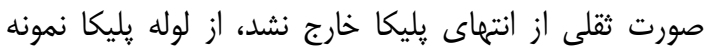

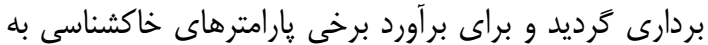

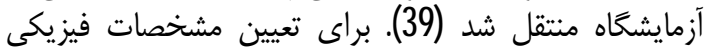

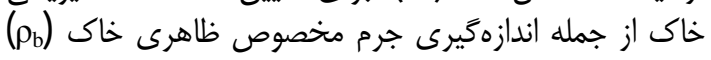

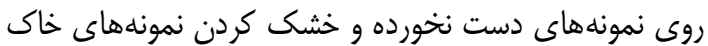

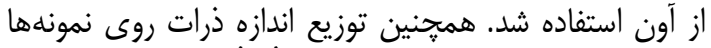

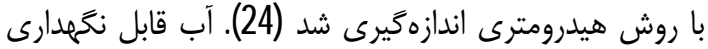

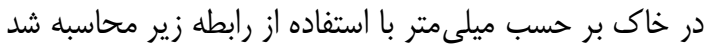

$\mathrm{AW}=\frac{\theta_{\mathrm{FC}}-\theta_{\mathrm{WP}}}{100} \times \mathrm{D} \times \frac{\rho_{\mathrm{b}}}{\rho_{\mathrm{w}}}$

اجزاى فرمول عبارتاند از AW آب قابل نتخهدارى

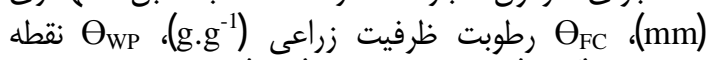

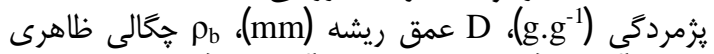
خاى (g.cm

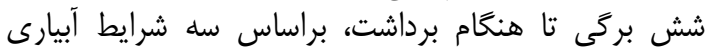

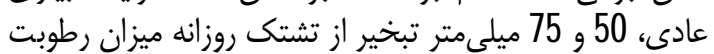

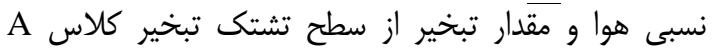

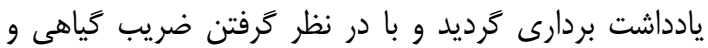

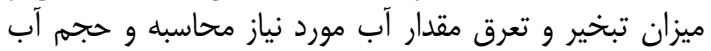

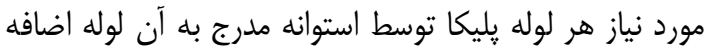

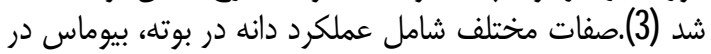

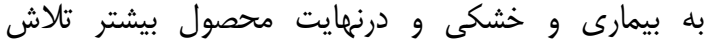

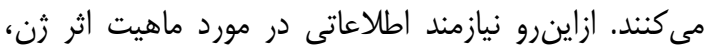

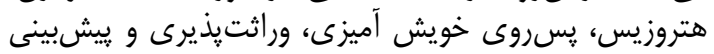

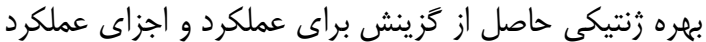

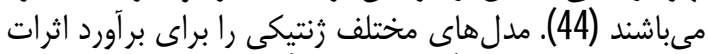

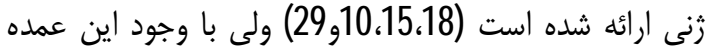

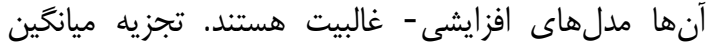

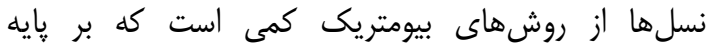

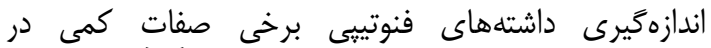
نسلهاى مختلف اصلاحى بنا شده است (25). در تجزئ دانيه

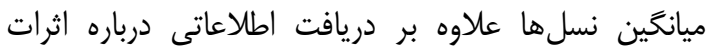

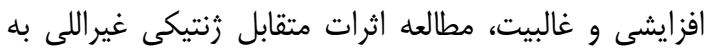

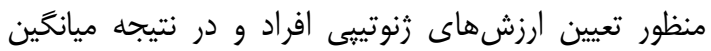

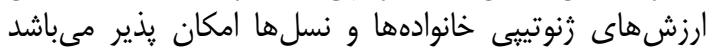

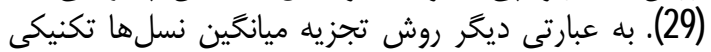

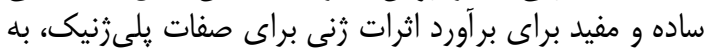



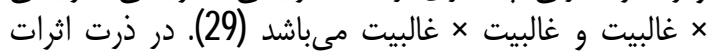

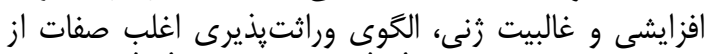

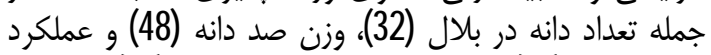

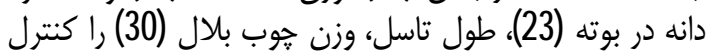

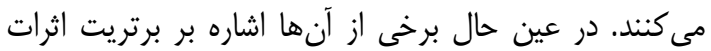

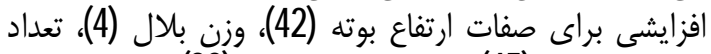

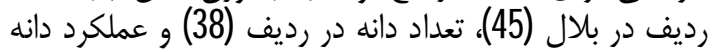

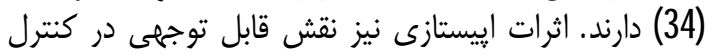

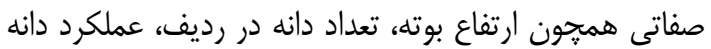

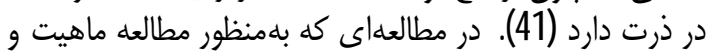

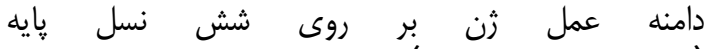

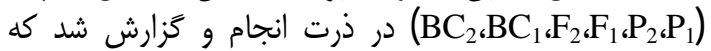

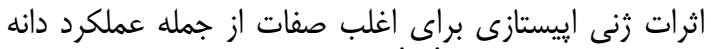

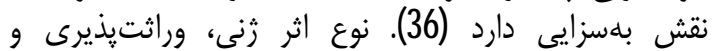

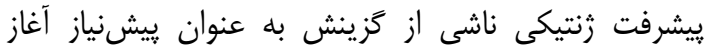

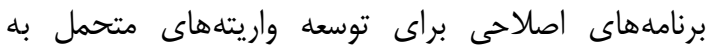

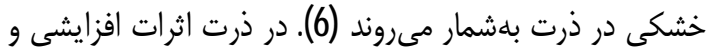

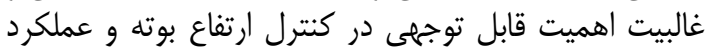

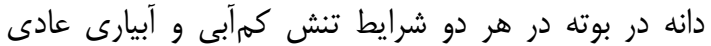

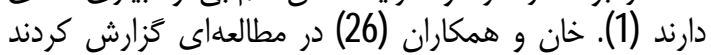

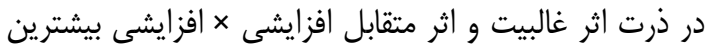

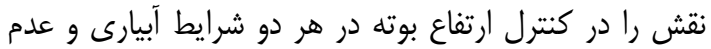

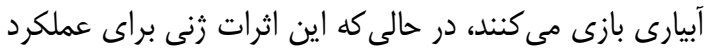

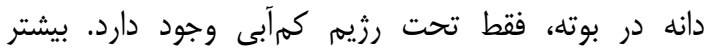

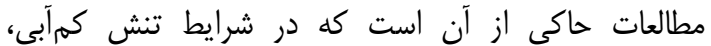

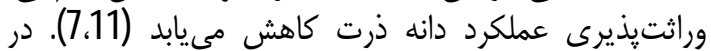

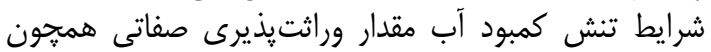

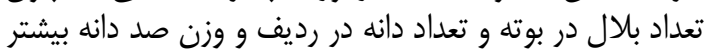

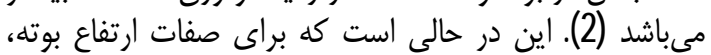

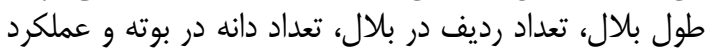

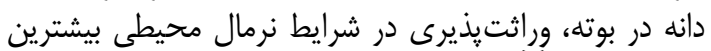

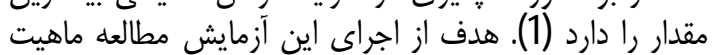


صحت مدل افزايشى - غالبيت توسط آزمون مقياس مورد

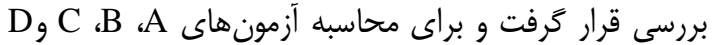

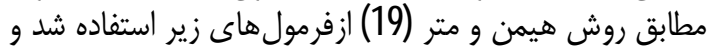

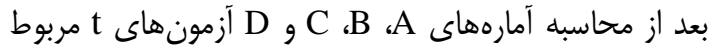

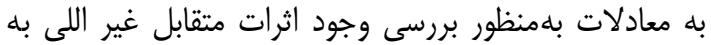
شرح زير برآورد شد:

$$
\begin{aligned}
& \mathrm{A}=2 \overline{\mathrm{BC}_{1}}-\overline{\mathrm{P}}_{1}-\overline{\mathrm{F}}_{1} \quad \mathrm{~V}_{\mathrm{A}}=4 \overline{\mathrm{V}}_{\mathrm{BC}_{1}}+\overline{\mathrm{V}}_{\mathrm{P}_{1}}+\overline{\mathrm{V}}_{\mathrm{F}_{1}} \\
& \mathrm{~B}=\overline{2 \mathrm{BC}_{2}}-\overline{\mathrm{P}_{2}}-\overline{\mathrm{F}_{1}} \quad \mathrm{~V}_{\mathrm{B}}=4 \overline{\mathrm{V}}_{\mathrm{BC}_{2}}+\overline{\mathrm{V}}_{\mathrm{P}_{2}}+\overline{\mathrm{V}}_{\mathrm{F}_{1}} \\
& \mathrm{C}=4 \overline{\mathrm{F}_{2}}-2 \overline{\mathrm{F}_{1}}-\overline{\mathrm{P}_{1}}-\overline{\mathrm{P}_{2}} \quad \mathrm{~V}_{\mathrm{C}}=16 \overline{\mathrm{V}}_{\mathrm{F}_{2}}+4 \overline{\mathrm{V}}_{\mathrm{F}_{1}}+\overline{\mathrm{V}}_{\mathrm{P}_{1}}+\overline{\mathrm{V}}_{\mathrm{P}_{2}} \\
& \mathrm{D}=4 \overline{\mathrm{F}}_{3}-2 \overline{\mathrm{F}_{2}}-\overline{\mathrm{P}}_{1}-\overline{\mathrm{P}}_{2} \quad \mathrm{~V}_{\mathrm{D}}=16 \overline{\mathrm{V}}_{\mathrm{F}_{3}}+4 \overline{\mathrm{V}}_{\mathrm{F}_{2}}+\overline{\mathrm{V}}_{\mathrm{P}_{1}}+\overline{\mathrm{V}}_{\mathrm{P}_{2}}
\end{aligned}
$$

$$
\begin{aligned}
\mathrm{SEA} & =\sqrt{\mathrm{V}_{\mathrm{A}}} & \mathrm{t}_{\mathrm{A}} & =\frac{\mathrm{A}}{\operatorname{SEA}} \\
\mathrm{SEB} & =\sqrt{\mathrm{V}_{\mathrm{B}}} & \mathrm{t}_{\mathrm{B}} & =\frac{\mathrm{B}}{\operatorname{SEB}} \\
\mathrm{SEC} & =\sqrt{\mathrm{V}_{\mathrm{C}}} & \mathrm{t}_{\mathrm{C}} & =\frac{\mathrm{C}}{\operatorname{SEC}} \\
\mathrm{SED} & =\sqrt{\mathrm{V}_{\mathrm{D}}} & \mathrm{t}_{\mathrm{D}} & =\frac{\mathrm{D}}{\operatorname{SED}}
\end{aligned}
$$

مقاديراجزاى واريانس زنتيكى يعنى واريانس هاى افزايشى

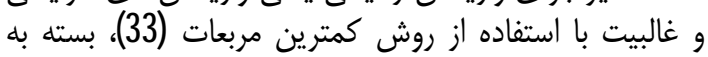

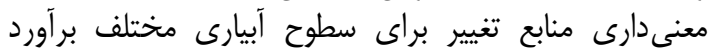

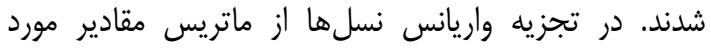

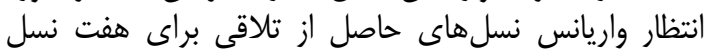

$$
\text { Observation }=\left[\begin{array}{c}
\overline{\mathrm{P}_{1}} \\
\overline{\mathrm{P}_{2}} \\
\overline{\mathrm{F}_{1}} \\
\overline{\mathrm{F}_{2}} \\
\overline{\mathrm{F}_{3}} \\
\overline{\mathrm{BC}_{1}} \\
\overline{\mathrm{BC}_{2}}
\end{array}\right] \quad \mathrm{X}=\left[\begin{array}{cccc}
1 & 0 & 0 & 0 \\
1 & 0 & 0 & 0 \\
1 & 0 & 0 & 0 \\
1 & 1 & 1 & 0 \\
1 & 1.5 & 0.75 & 0 \\
1 & 0.5 & 1 & -1 \\
1 & 0.5 & 1 & 1
\end{array}\right]
$$

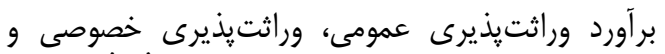

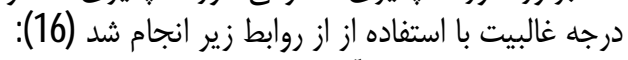



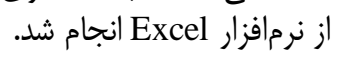

$$
\begin{aligned}
& \mathrm{h}_{\mathrm{b}}^{2}=\frac{\mathrm{V}_{\mathrm{A}}+\mathrm{V}_{\mathrm{D}}}{\mathrm{V}_{\mathrm{A}}+\mathrm{V}_{\mathrm{D}}+\frac{\mathrm{V}_{\mathrm{E}}}{\mathrm{r}}} \\
& \text { وراثتيذيرى عمومى } \\
& \mathrm{h}_{\mathrm{n}}^{2}=\frac{\mathrm{V}_{\mathrm{A}}}{\mathrm{V}_{\mathrm{A}}+\mathrm{V}_{\mathrm{D}}+\frac{\mathrm{V}_{\mathrm{E}}}{-}} \quad \text { gواثتيذيرى خصوصى } \\
& \overline{\mathrm{a}}=\sqrt{\frac{2 \mathrm{~V}_{\mathrm{D}}}{\mathrm{V}_{\mathrm{A}}}} \quad \quad \quad \text { درجه غالبيت }
\end{aligned}
$$

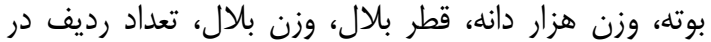

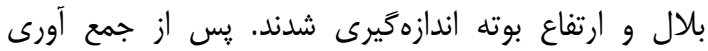

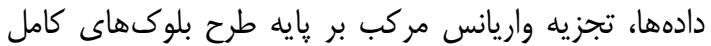

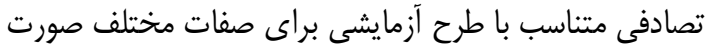

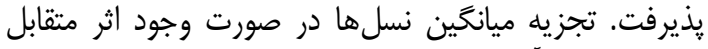

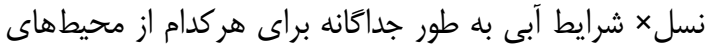

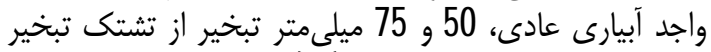

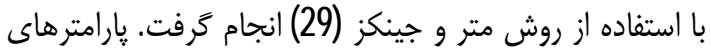

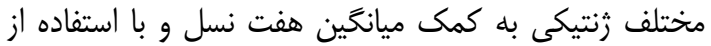
روش كمترين مربعات وزنى برآورد شد. اجزاى تشكي تشكيلدهنده ميانكين كل هر صفت:

$Y=m+\alpha[a]+\beta[d]+\alpha^{2}[i]+2 \alpha \beta[j]+\beta^{2}[l]$

اجزاى فرمول عبارتاند از Y ميانكين يك نسل،

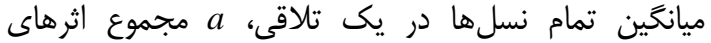

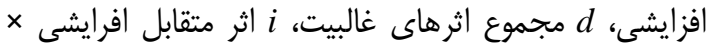

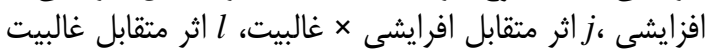

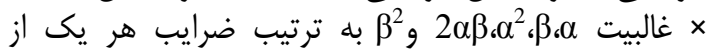

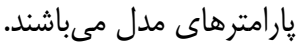
$\beta=\left(X^{\prime} W X\right)^{-1}$. $\left(X^{\prime} W Y\right)$

در اين روش وزن هر نسل عبارت است از (29): $\mathrm{W}=\frac{1}{\frac{\mathrm{V}}{\mathrm{n}}}$

Estimation $=\mathrm{X} \hat{\beta}$

$\chi^{2}=\sum\left[\left(\mathrm{O}_{\mathrm{i}}-\mathrm{E}_{\mathrm{i}}\right)^{2} \cdot \mathrm{W}\right]$

معنى دار بودن مقادير پارامترهاى برآورد شده نيز از طريق

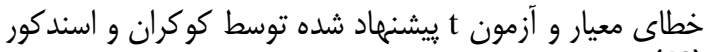
(43) تعريف شده صورت كر فت.

$\mathrm{SE}=\left(\left(\mathrm{X}^{\prime} \mathrm{WX}\right)^{-1}\right)^{0.5}$

افزون بر اين از طريق آزمون مقياس مشترك و با استفاده از از

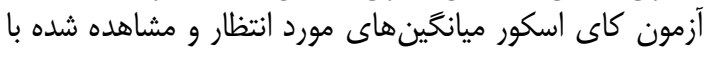

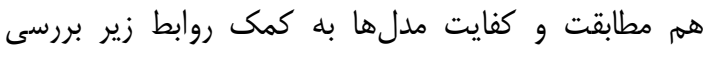

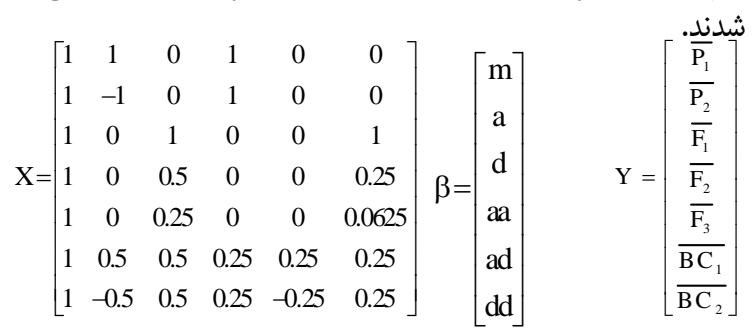
$\mathrm{W}=\left[\begin{array}{ccccccc}\mathrm{W}_{\mathrm{P}_{1}} & 0 & 0 & 0 & 0 & 0 & 0 \\ 0 & \mathrm{~W}_{\mathrm{P}_{2}} & 0 & 0 & 0 & 0 & 0 \\ 0 & 0 & \mathrm{~W}_{\mathrm{F}_{1}} & 0 & 0 & 0 & 0 \\ 0 & 0 & 0 & \mathrm{~W}_{\mathrm{F}_{2}} & 0 & 0 & 0 \\ 0 & 0 & 0 & 0 & \mathrm{~W}_{\mathrm{F}_{3}} & 0 & 0 \\ 0 & 0 & 0 & 0 & 0 & \mathrm{~W}_{\mathrm{BC}_{1}} & 0 \\ 0 & 0 & 0 & 0 & 0 & 0 & \mathrm{~W}_{\mathrm{BC}_{2}}\end{array}\right]$ 
نشان از تاثير تنش كما آبى بر عملكرد دانه در بوته، بيوماس در



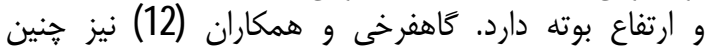

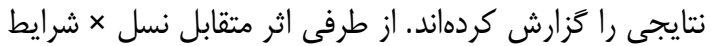

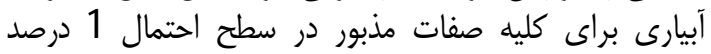

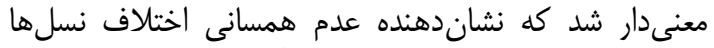

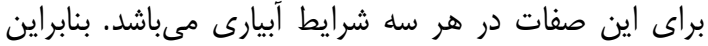

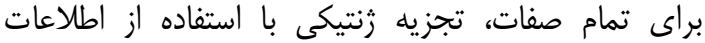

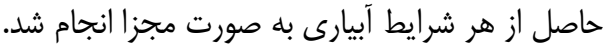

\section{نتايج و بحث تجزيه واريانس مركب}

با توجه به نتايج تجزيه واريه واريانس مركب بران براى صفات

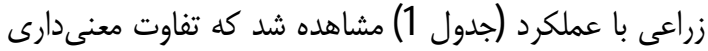

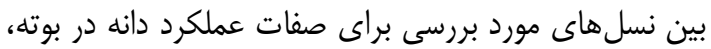

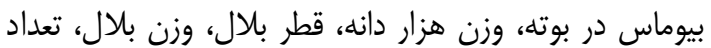

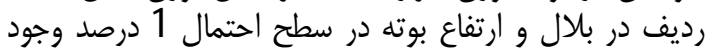

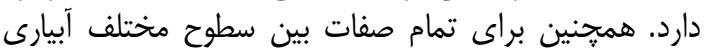
اختلاف معنى دارى در سطح احتمال 1 درصد وجود داشت كمات كه

جدول 1 - تجز يه واريانس مركب صفات زراعى تحت شرايط آبيارى عادى و50 و5 ميلى متر تبخير از تشتى كلاس Table 1. Combined variance analysis of agronomic traits under normal irrigation, 50 and $75 \mathrm{~mm}$ evaporation from class A evaporation pan

\begin{tabular}{|c|c|c|c|c|c|c|c|c|}
\hline \multicolumn{7}{|c|}{ ميانگين مربعات } & \multirow{2}{*}{ آزادى } & \multirow[b]{2}{*}{ منابع تغييرات } \\
\hline ارتفاع بوته & تعداد رديف & وزن بلال & قطر بلال & وزن هزاردانه & بيوماس در بوته & $\begin{array}{c}\text { عملكرد دانه در } \\
\text { بوته }\end{array}$ & & \\
\hline $55302 / 215^{* *}$ & $185 / 360^{* *}$ & $111846903^{* *}$ & $91 / 107^{*}$ & $3343 / 328^{* *}$ & $303712127^{* *}$ & $123955 / 409^{*}$ & 2 & شرايط آبيارى \\
\hline 291/698 & 4555 & $708 / 591$ & व163 & 29025 & 1284724 & $541 / 055$ & 57 & تكرار / شرايط \\
\hline $226 / 1108^{* *}$ & $43 / 406^{* *}$ & $38302 / 243^{* *}$ & $4591^{* *}$ & $147 / 852^{* *}$ & $6855 / 651^{* *}$ & $37830856 *$ & 6 & نسل \\
\hline $67 / 82^{* *}$ & $2 / 123^{* *}$ & $3746868^{* *}$ & $002^{* *}$ & $9 / 489^{* *}$ & $48 / 3 / 343^{* *}$ & $2794279^{* *}$ & 12 & نسل× شرايط آبيارى \\
\hline $67 / 822$ & 3655 & 474170 & Q305 & $4 / 181$ & $1414 / 866$ & $492 / 513$ & 34 & خطا \\
\hline $10 / 7$ & $16 / 16$ & 15/8 & $16 \propto$ & $\varepsilon / 2$ & $16 / 9$ & $1 \varepsilon / 4 E$ & & ضريب تغييراتهو) \\
\hline
\end{tabular}

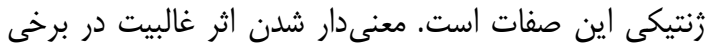

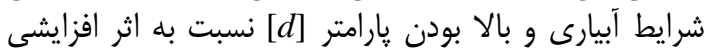

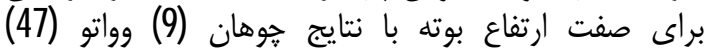

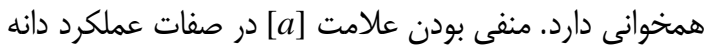

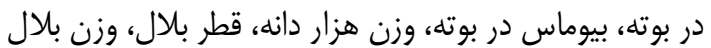

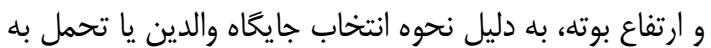

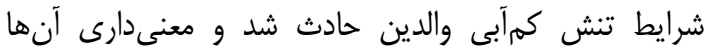

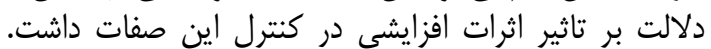

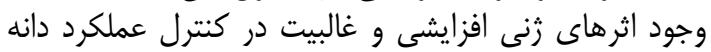

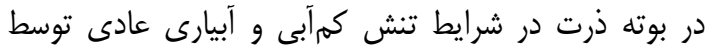

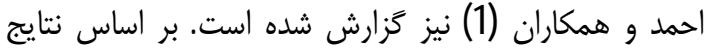

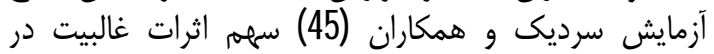

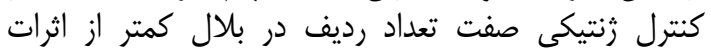

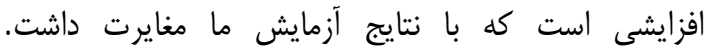

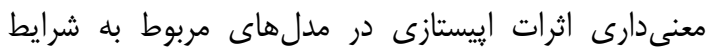

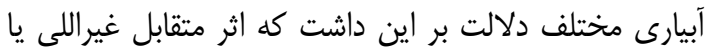

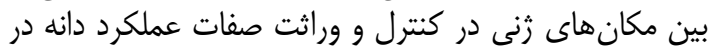



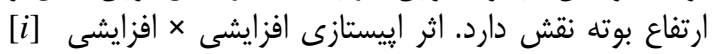

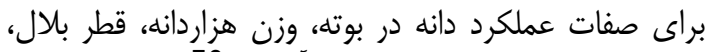

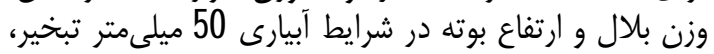

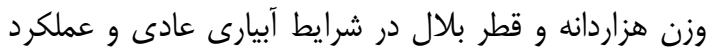

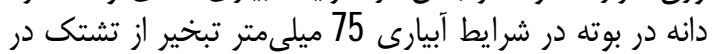

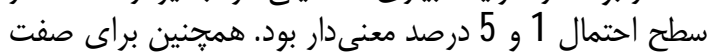

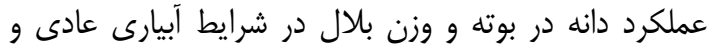

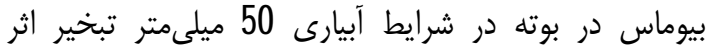
إييستازى افزايشى X غالبيت [j] در سطح احتمال 1 و5 درئر درصد
براساس نتايج تجزيه زينتيكى با استفاده از آزمون كفايت

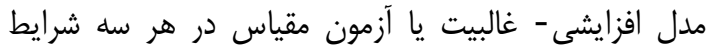

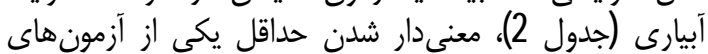

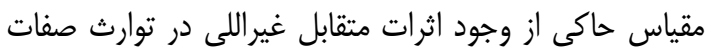

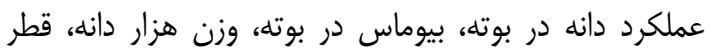

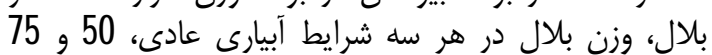

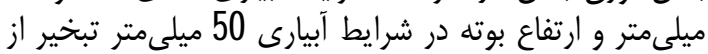

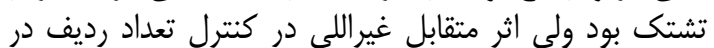

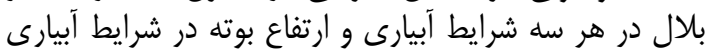
عادى و50 ميلى متر تبخير نقش نداشت.

تجزيه ميانكَين نسلها ماديا

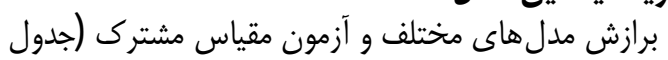

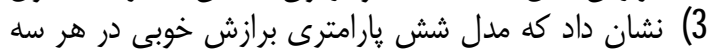

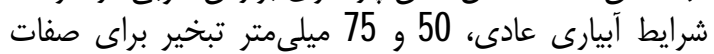

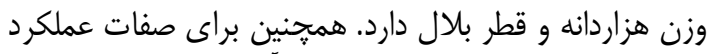

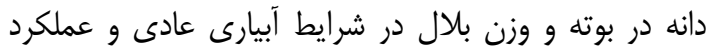

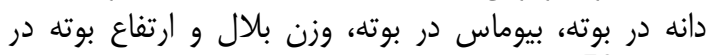

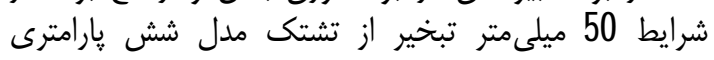

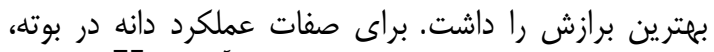

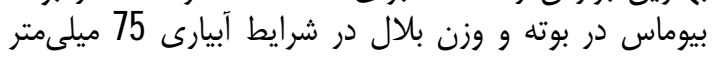

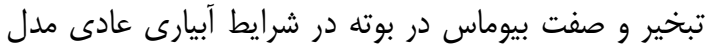

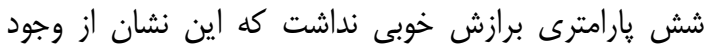

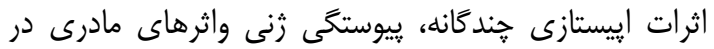

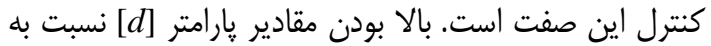

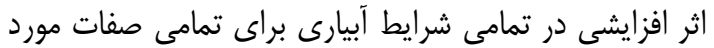
مطالعه نشان دهنده سعهم موثر اثر غالبيت ثرائى در در كنترل 
بيوماس در بوته، وزن هزار دانه، وزن بلال و قطر بلال

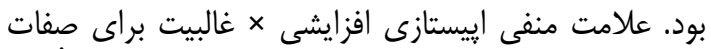

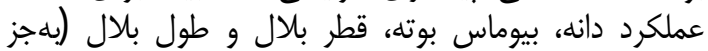



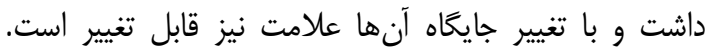

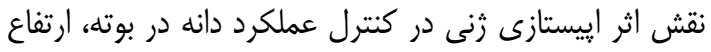

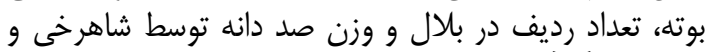
همكاران (40) نيز كزارش شده است.

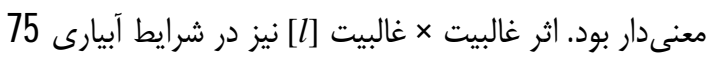

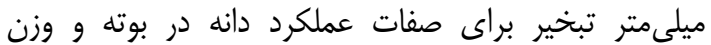

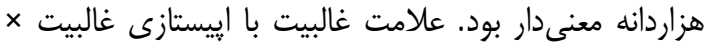

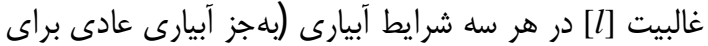

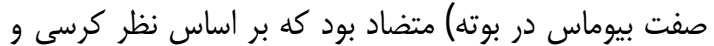

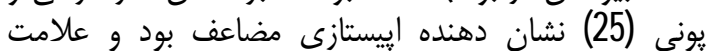

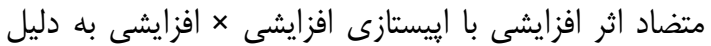
ماهيت متضاد اثر متقابل در صفات عملكرد دانه در بوتئها إنها

جدول2 - آزمون كفايت مدل افزايشى - غالبيت براى صفات زراعى تحت شرايط آبيارى عادى و50 و \$ ميلىمتر تبخير از تشتك كلاس A Table 2. Scaling test for agronomic traits under normal irrigation, 50 and $75 \mathrm{~mm}$ evaporation from class A

\begin{tabular}{|c|c|c|c|c|}
\hline \multicolumn{3}{|c|}{ شرايط أبيارى } & \multirow{2}{*}{ مقياسها } & \multirow{2}{*}{ صفات } \\
\hline T27 ميلى متر & SC ميلىمتر & عادى & & \\
\hline $1 / 8 \& \pm C / 7 \varepsilon^{n s}$ & $2=1 \pi \pm 12 / 4)^{\mathrm{ns}}$ & $-50 / 11 \pm 15 / 17$ & $\mathrm{~A}$ & \multirow{4}{*}{ عملكرد دانه در بوته } \\
\hline $1 / 5 \pm 1 C / 91^{\mathrm{ns}}$ & $2 d / 15 \pm 12 / 0$ & $2 \varepsilon / 0 \epsilon \pm 1 \varepsilon / 4 \epsilon^{\mathrm{ns}}$ & B & \\
\hline $120 / 84 \pm 20 / 82$ & $8 / 2 \varepsilon+24 / 45$ & $\bar{x} / 0= \pm 3 \mathrm{C} / 0 \mathrm{c}^{\mathrm{ns}}$ & $\mathrm{C}$ & \\
\hline$-74 / 8 \pm 15 / 0 \%$ & $41 / T \pm \mathbb{C} / 3 C$ & $-55 / 4] \pm 2 c / 6$ & D & \\
\hline$-4 / \lambda \pm 13 / T^{11 \mathrm{II}}$ & $-c / 2 \pm 1 E / Q^{1 I S}$ & $-\sigma / 7 L \pm 2 \delta / 3 c$ & $\mathrm{~A}$ & \multirow{4}{*}{ بيوماس در بوته } \\
\hline$-4 / 8 \pm 14 / 4 \varepsilon^{n s}$ & $4 c / 6] \pm 17 / 3]$ & $-4=/ 9 E+2 E / 1^{n s}$ & B & \\
\hline $15 / / T \pm \pm 3 A / 4 C^{\pi \cdot T}$ & $3 / 117 \pm 3 c / 47^{n s}$ & 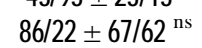 & $\mathrm{C}$ & \\
\hline$-111 / \sigma \pm 2 c / \pi c$ & $26 / T_{L} \pm 32 / 5 C^{\mathrm{ns}}$ & $-196 / 3 \angle \pm 5 c / 0=$ & D & \\
\hline$C / T_{L} \pm C / \sigma^{n S}$ & $1 / T= \pm 1 / 5^{1 \mathrm{nS}}$ & $\exists / 9 E \pm 1 / 76$ & A & \multirow{4}{*}{ وزن هزاردانه } \\
\hline$-\left(/ 5= \pm C / 64^{n s}\right.$ & $-1 / 2 \pm \pm 1 / 5 c^{n s}$ & $-\left(/ O A \pm 1 / 74^{\mathrm{ns}}\right.$ & B & \\
\hline - 派 $56 \pm 1 / 35$ & 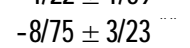 & $-5 / 94 \pm \equiv / 944^{\mathrm{ns}}$ & $\mathrm{C}$ & \\
\hline$-2 / 7 / \pm 1 / \sigma^{n s}$ & $-6 / 4 \pm \pm \equiv / 8^{n s}$ & $-z / 9 \pm \equiv / g^{\mathrm{ns}}$ & D & \\
\hline$-(12 x \pm C / 3]^{115}$ & $-\left(/ 3^{5} \pm C / 3\right]^{11 s}$ & $-\left(/ 3^{5} \pm C / 2^{11 s}\right.$ & $\mathrm{A}$ & \multirow{4}{*}{ قطر بلال } \\
\hline$-C / 2 \pm C / 3 C^{n s}$ & $-C / 11 \pm C / 2 c^{n s}$ & $-C / X \pm C / Z^{\mathrm{ns}}$ & B & \\
\hline$-(/ 81 \pm C / 6 /)^{n s}$ & $-C / 5^{5} \pm C / 6 C^{n s}$ & $-C / 7] \pm C /[]^{n s}$ & $\mathrm{C}$ & \\
\hline$-1 / 17 \pm C / 5 C$ & $-1 / 4= \pm C / 5$ & $-1 / 3 x \pm C / 4 /$ & D & \\
\hline $38 / 14 \pm c / 2 i$ & $1 / \propto \pm \pm 12 / 9 E^{n s}$ & $-3 d / 16 \pm 14 / 74$ & A & \multirow{4}{*}{ وزن بلال } \\
\hline $5 / \& \pm 1 C / 04^{n s}$ & $21 / 4 \pm 11 / 64^{n s}$ & $-3 C / 7 C \pm 14 / 0 C^{-\pi}$ & B & \\
\hline $10 / 94 \pm 22 / 71$ & $\& \mathscr{C} / \mathrm{TE} \pm \mathrm{Z} / \mathrm{T}$ & $2 E / 1 \angle \pm 3=/ T 1^{\mathrm{ns}}$ & $\mathrm{C}$ & \\
\hline$-45 / 91 \pm 17 / 6$ & $2 / 54 \pm 17 / 3 c^{\mathrm{ns}}$ & $-5 / / 36 \pm 2 / \alpha^{n s}$ & $\mathrm{D}$ & \\
\hline$-\left(/ \bar{C} \pm 1 / 1 z^{\mathrm{ns}}\right.$ & $C / \equiv \pm 1 / O E^{115}$ & $-1 / \equiv \pm C / 9]^{1 \mathrm{nS}}$ & A & \multirow{4}{*}{ تعداد رديف در بلال } \\
\hline$-1 / O \pm 1 / 1^{n s}$ & $-C / \mathcal{E} \pm 1 / 1^{\mathrm{ns}}$ & $-C / \subseteq \pm C / G^{\mathrm{ns}}$ & B & \\
\hline$y / \subseteq \pm 2 / 2^{n s}$ & $2 / \equiv \pm 2 / 2^{\mathrm{ns}}$ & $C /] \pm 1 / \mathscr{C}^{\mathrm{ns}}$ & $\mathrm{C}$ & \\
\hline $1 / \varepsilon \pm 1 / 9 E^{\mathrm{ns}}$ & $\Xi_{1} \pm 2 / 1 \mathrm{c}^{\mathrm{ns}}$ & $-C / 5 \pm 1 / \mathbb{E}^{\mathrm{ns}}$ & D & \\
\hline $7 / 0046 / 52^{1 \mathrm{IS}}$ & $2 / 4 \mathrm{z} \pm \varepsilon / 9^{\mathrm{ns}}$ & $1\left(/ 2 \epsilon \pm \varepsilon / 1 \epsilon^{1 \mathrm{~ns}}\right.$ & A & \multirow{4}{*}{ ارتفاع بوته } \\
\hline$\epsilon / \mathbb{C}^{E} \pm \epsilon / 4^{\mathrm{ns}}$ & $C / 56 \pm 1 C / 7 c^{\mathrm{ns}}$ & $-(/ 5 x \pm 7 / 6]^{n s}$ & B & \\
\hline $4 / 1 \pm \pm 15 / \mathscr{C}^{\mathrm{ns}}$ & $25 / 64 \pm 2 C / 3^{n s}$ & $-16 / 0 \pm 21 / 94^{\mathrm{ns}}$ & $\mathrm{C}$ & \\
\hline $20 / 6 / \pm 15 / 74^{n s}$ & $4 C / 9 \pm \pm 14 / 6 C$ & $16 / 4^{2} \pm 1 \varepsilon / 3^{\mathrm{ns}}$ & D & \\
\hline
\end{tabular}


جدول3 - يارامترهاى زنتيكى و آزمون مقياس مشترى براى صفات زراعى از طريق تجزيه ميانكين نسل ها تحت شرايط آبيارى عادى و50 و A

Table 3. Genetic parameters and joint scaling test for agronomic traits by generation mean analysis under normal irrigation, 50 and $75 \mathrm{~mm}$ evaporation from class A evaporation pan

\begin{tabular}{|c|c|c|c|c|c|c|c|c|}
\hline \multirow{2}{*}{$\chi^{2}(\mathrm{df})$} & \multicolumn{6}{|c|}{ بإرامترهاى زنتيكى } & \multirow{2}{*}{ شرايط آبيارى } & \multirow{2}{*}{ صفات } \\
\hline & {$[l]$} & [j] & {$[i]$} & {$[d]$} & {$[a]$} & $m$ & & \\
\hline $3 / 34(1)^{\mathrm{ns}}$ & $-2 / 69 \pm 30 / 46^{\text {ns }}$ & $-80 / 21 \pm 2004^{* *}$ & $11 / 00 \pm 11 / 91^{\mathrm{ns}}$ & $87 / 18 \pm 37 / 85^{*}$ & $-5 / 94 \pm 3 / 99^{\mathrm{ns}}$ & $114 / 89 \pm 11 / 71^{* *}$ & عادى & عملكرد \\
\hline $\begin{array}{l}C / \mathbb{C}(1]^{\text {IIs }} \\
26 / 1(1)^{* *}\end{array}$ & $\begin{array}{l}-34 / T \pm \pm 24 / \mathbb{O}^{\mathrm{IIS}} \\
-74 / 7 \pm \pm 194^{* *}\end{array}$ & $\begin{array}{l}-4 / 2 \pm \pm 15 / 1 \varepsilon^{115} \\
-3 / 64 \pm 12 / 85^{\text {ns }}\end{array}$ & $\begin{array}{l}-11 / 64 \pm \varepsilon / \Subset \\
18 / 35 \pm 6 T^{* *}\end{array}$ & $\begin{array}{l}86 / 17 \pm 2 \varepsilon / \& \\
132 / 89 \pm 23 / 53^{* *}\end{array}$ & $\begin{array}{l}-7 / 2 \pm z / 2 \\
-19 / 66 \pm 1 / 95^{* *}\end{array}$ & $\begin{array}{l}96 / \propto \pm \pm \varepsilon / 5 \varepsilon^{*} \\
53 / 90 \pm 6 / \sigma^{* *}\end{array}$ & T 5 ميلى متر 5 متر & د د د دوته \\
\hline $1066(1)^{* *}$ & $56 / 79 \pm 47 / 40^{\text {ns }}$ & $-16 / 10 \pm 32 / 06^{\text {ns }}$ & $22 / 3 \pm 21 / 97^{\mathrm{ns}}$ & $65 / 54 \pm \lll / 50^{\text {ns }}$ & $-15 / 99 \pm 7 / 57^{*}$ & $22 / 30 \pm 6127^{* *}$ & 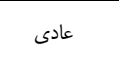 & \\
\hline $\begin{array}{l}C / O O E\left[(1)^{113}\right. \\
2 E / 3(1)\end{array}$ & $\begin{array}{l}-21 / 54 \pm 3 z / G^{113} \\
-1 C / 8 / \pm 2 \pi / 99^{113}\end{array}$ & $\begin{array}{l}-4 \varepsilon / \pi \pm 22 / 3 x \\
-\left(/ 5 / \pm 1 / 1 / 1^{115}\right.\end{array}$ & $\begin{array}{c}-\varepsilon / 6 / 1 \pm 1 \equiv / 5 S^{11 \mathrm{~s}} \\
4 / 9 \mathrm{C} \pm 1 C / 9]^{\mathrm{II}} \\
\end{array}$ & $\begin{array}{l}9 / 5 x \pm 41 / 6 \\
96 / 2 x \pm 3=/ 8\end{array}$ & $\begin{array}{c}C / 21 \pm 4 / 3^{11} \\
-2 E / 2 E \pm \equiv / 64 \\
\end{array}$ & $\begin{array}{l}177 / \in G \pm 1=/ 3 \\
14 / / 31 \pm 1 / 74\end{array}$ & 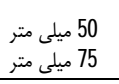 & لدر بوته \\
\hline $1 / \mathscr{g}(1)^{\mathrm{ns}}$ & $-7 / 93 \pm 3 / 50^{\mathrm{ns}}$ & $3 / 99 \pm 2 / 23^{\mathrm{ns}}$ & $4 / 92 \pm 1 / 64^{* *}$ & $15 / \Omega_{ \pm} \pm 464^{*}$ & $-1 / 57 \pm 0156^{\mathrm{ns}}$ & $23 / 94 \pm 1 / \Omega^{* *}$ & عادى & \\
\hline $\begin{array}{l}1 / 4 \mathbb{E}(1]^{\mathrm{us}} \\
1 / 2(1)^{\mathrm{IIs}}\end{array}$ & $\begin{array}{l}-5 / 1 \pm \pm \equiv / 1 \epsilon^{115} \\
-2 / 1 \epsilon \pm 1 / 3\end{array}$ & $\begin{array}{l}2 / 97 \pm 2 / 1={ }^{-13} \\
1 / 26 \pm(/ 8)^{\text {"is }}\end{array}$ & $\begin{array}{l}5 / 5 C \pm 1 / 6 \\
2 / 3 C \pm C / 6^{13}\end{array}$ & $\begin{array}{l}13 / 9 \pm \pm 4 / 5]^{115} \\
5 / 7 \pm \pm 1 / 8\end{array}$ & $\begin{array}{l}-1 / 91 \pm(/ 4) \\
-C / 7 \subseteq \pm C / 1 C\end{array}$ & $\begin{array}{l}1 \varepsilon / 9= \pm 1 / 6 \\
17 / 4= \pm(/ 6)\end{array}$ & TE ميلى TCيلى متر & هزاردانه \\
\hline $0 / 46(1)^{\mathrm{ns}}$ & $-\alpha 10 \pm \alpha / 48^{\text {ns }}$ & $-\alpha 12 \pm \alpha 36^{\mathrm{ns}}$ & $0158 \pm 019^{* *}$ & $1 / 01 \pm \alpha Q^{\mathrm{ns}}$ & $-Q / 30 \pm Q 0 B^{* *}$ & $3 / 69 \pm 018^{* *}$ & عادى ل ع ل ع & ق. \\
\hline $\begin{array}{l}\mathrm{C} / 5 /(1]^{\mathrm{\mu s}} \\
\mathrm{C} / \mathrm{OE}(1)^{\mathrm{\mu s}}\end{array}$ & $\begin{array}{l}-C / 2 \pm C / 54^{110} \\
-C / 17 \pm C / 5)^{118}\end{array}$ & 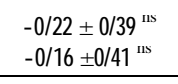 & $\begin{array}{l}C / 5 C \pm C / 27 \\
C / 5 C \pm\left(/ 22^{113}\right.\end{array}$ & $\begin{array}{l}1 / 32 \pm C / \pi^{11} \\
1 / 26 \pm C / 7]\end{array}$ & $\begin{array}{l}-C / 1 C \pm C / \propto \\
-C / 2 C \pm C / \propto \\
\end{array}$ & $\begin{array}{l}2 / 7] \pm C / 22 \\
2 / 01 \pm C / 2\end{array}$ & 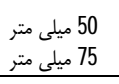 & ل الال \\
\hline $1 / 48(1)^{\mathrm{ns}}$ & $-24 / 97 \pm 28 / 80^{n s}$ & $-77 / 49 \pm 17 / 94^{* *}$ & $17 / 08 \pm 12 / 30^{\mathrm{ns}}$ & $124 / 90 \pm 3651^{* *}$ & $-2 / 25 \pm 3 / 87^{n s}$ & $120156 \pm 27 / 14^{* *}$ & 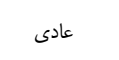 & \\
\hline $\begin{array}{l}1 / 8=[1])^{\mu \mathrm{m}} \\
1 \mathrm{C} / 1(1)\end{array}$ & 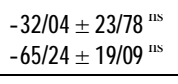 & $\begin{array}{l}-1 / 14 \pm 14 / 74^{115} \\
3 C / 84 \pm 11 / \Re^{115}\end{array}$ & $\begin{array}{l}-15 / \propto \pm \pm] / 56 \\
1[/ 24 \pm 7 / 3]^{115} \\
\end{array}$ & $\begin{array}{c}9 E / 02 \pm 26 / 91 \\
10 / 36 \pm 2=18 \\
\end{array}$ & $\begin{array}{r}-7 / 56 \pm 2 / 6 \\
-17 / 15 \pm 2 / x \\
\end{array}$ & $\begin{array}{l}108 / 51 \pm 7 / 4 C \\
8 / 17 \pm 7 / 2\end{array}$ & 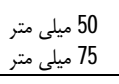 & بلال \\
\hline $2 / 64(4)^{\mathrm{ns}}$ & - & - & - & $2 / 50 \pm 0 / 45^{* *}$ & $\alpha Q_{ \pm} \alpha 24^{*}$ & $12 / 14 \pm Q 23^{* *}$ & 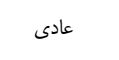 & تعداد \\
\hline $\begin{array}{l}E / 1 / 4(4)^{\text {nis }} \\
5 / T(4)^{\text {II }}\end{array}$ & $\begin{array}{l}- \\
-\end{array}$ & $\begin{array}{l}- \\
-\end{array}$ & $\begin{array}{l}- \\
-\end{array}$ & $\begin{array}{l}2 / 2= \pm C / 5 C \\
1 / 6 \pm C / 5\end{array}$ & $\begin{array}{l}C / 3 \pm \pm(/ 2)^{115} \\
C / 15 \pm(/ 25\end{array}$ & $\begin{array}{l}1 / / 51 \pm C / 2 E \\
1 C / 3 C \pm C / 2\end{array}$ & 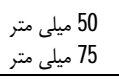 & در بلال \\
\hline $3 / 03(4)^{\mathrm{ns}}$ & - & - & - & $14 / 20 \pm 4 / 55^{* *}$ & $5 / 49 \pm 2 / 04^{* *}$ & $16 / 29 \pm 2 / 09^{* *}$ & عادى & ارت \\
\hline 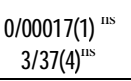 & $\begin{array}{c}1 \mathcal{C} / 9 \overline{9 \top} \pm 1 \in / Z^{\mathrm{IS}} \\
-\end{array}$ & $\begin{array}{c}1 / 8 \pm \pm 11 / 9^{11 \mathrm{~s}} \\
-\end{array}$ & $\begin{array}{c}-2 / 9 x \pm 6 / 17 \\
-\end{array}$ & $\begin{array}{c}-21 / 91 \pm 20 / 8]^{115} \\
14 / 8 \pm \pm \equiv / 3 E^{15}\end{array}$ & $\begin{array}{l}-c / 01 \pm \equiv / x \\
-\equiv / 9 \pm \pm 1 / 56\end{array}$ & $\begin{array}{l}16 / 1 \varepsilon \pm 5 / \& \\
12 / 0 \pm \pm 1 / 56\end{array}$ &  & بوته \\
\hline
\end{tabular}

درجه غالبيت به دليل منفى شدن واريانس غالبيت و صفر

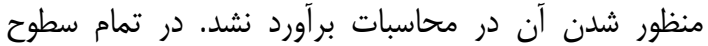



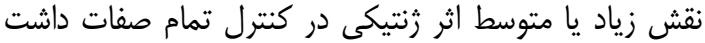

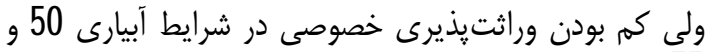

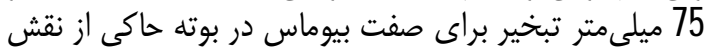

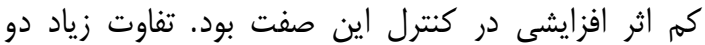
وراثتيذيرى عمومى و خصوصى در ائى شرايط آبيارى 50 و 75

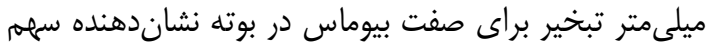

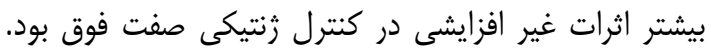

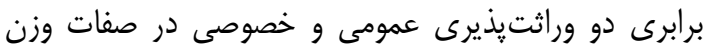

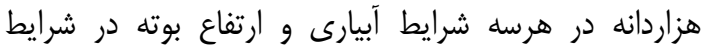

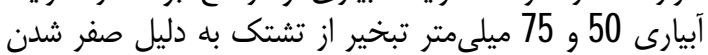

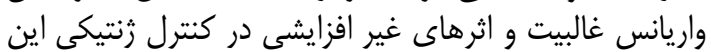

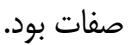

\section{تجزيه واريانس نسلها} نتايج تجزيه واريانس نسلهانها (جدول 4) نشان دانس داد كه

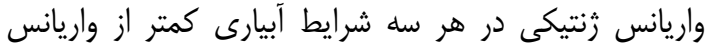

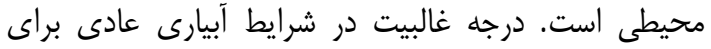

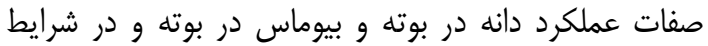

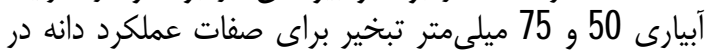

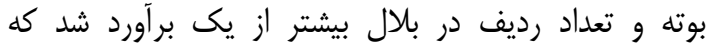

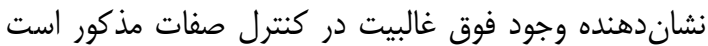

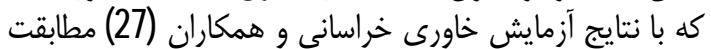

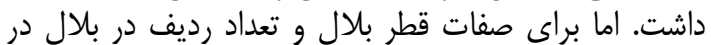

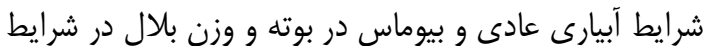

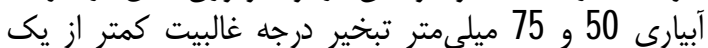

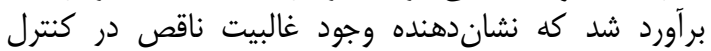

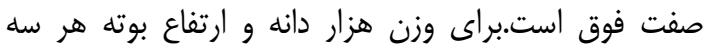
شرايط آبيارى و صفت وزن بلال در شرايط آبيارى نرمال 
جدول4 - اجزاى واريانس زنتيكى، وراثتيذيرى عمومى، وراثت يذيرى خصوصى و درجه غالبيت براى صفات زراعى تحت شرايط آبيارى عادى A

Table 4. Estimation variance components, broad-sense heritability, narrow-sense heritability and degree of dominance for agronomic traits under normal irrigation, 50 and $75 \mathrm{~mm}$ evaporation from class A evaporation pan

\begin{tabular}{|c|c|c|c|c|c|c|c|}
\hline \multicolumn{2}{|c|}{ وراثتيذيرى } & \multirow{2}{*}{ درجه غالبيت } & \multicolumn{3}{|c|}{ اجزاى واريانس زرنتيكى } & \multirow{2}{*}{ شرايط آبيارى } & \multirow{2}{*}{ صفات } \\
\hline خصوصى & عمومى & & واريانس محيطى & واريانس غالبيت & واريانس افزايشى & & \\
\hline- & 8432 & $2 / 11$ & $75 / 26$ & $20 / 31$ & - & عادى & \\
\hline - & $9 / 4 C$ & Z/E & $36-136$ & $260 / 6$ & - & 5C ميلى متر & عملكرد دانه \\
\hline- & 9/1C & 20,64 & $26 / 8$ & $2 \beth=/ 11$ & - & T Tيلى متر & \\
\hline $56 / \pi$ & $91 / 51$ & $1 / 9 E$ & TSEव & $3 / / 4]$ & 69E/9E & عادى & \\
\hline $1 / \mathbb{C}^{E}$ & $86 / 6$ & $C / 2 C$ & $825 / 4 C$ & $26=/ 04$ & $5 / \pi$ & CC ميلى متر & بيوماس \\
\hline$C / 2 C$ & $58 / 3 C$ & $C / O E$ & $69 / 2$ & $4 \varepsilon / \propto$ & $\mathrm{C} / 17$ & T Tيلى متر & \\
\hline $8 / / 85$ & $8 / / 85$ & - & $12 / 4 E$ & - & $4 / 4 E$ & عادى & \\
\hline $9=/ 31$ & $9=/ 31$ & - & $7 / 15$ & - & $5 / 04$ & CC ميلى متر & وزن هزاردانه \\
\hline $9 / \sqrt{3}=$ & $9 / 3=$ & - & $1 / 24$ & - & $1 / 0 E$ & T2T ميلم متر & \\
\hline - & $90 / 6=$ & C/4A & $C / 2$ & C/IC & - & عادى & \\
\hline - & $9 / 6$ & $\mathrm{C} / 5 \mathrm{C}$ & $\mathrm{C} / 24$ & $\mathrm{C} / 17$ & - & C 5 ميلى متر & قطر بلال \\
\hline- & $9 / / 02$ & $c / .6=$ & $C / 2 C$ & $C / 2 C$ & - & TL ميلى متر & \\
\hline$\pi / 3 x$ & $T / 3 E$ & - & $70 / 11$ & - & $11 C / 76$ & عادى & \\
\hline - & $8 \mathbb{8} / 5$ & $1 / / 92$ & $46 / / \Subset$ & $17 / 99$ & - & 5C ميلى متر & وزن بلال \\
\hline - & $8 / 2$ & 12/85 & $286 / 6$ & $8 / 6$ & - & TL ميلى متر & \\
\hline- & $5=18=$ & $\mathrm{C} / 5 \mathrm{C}$ & $3 / 02$ & C/17 & - & عادى & \\
\hline- & $84 / 3 C$ & $1 / 4$ & $\Xi / 6$ & C/GE & - & CC ميلى متر & تعداد رديف \\
\hline - & $8=/ 56$ & $1 / 4$ & $4 / 05$ & $1 / 04$ & - & T2 ميلى متر & در بلال \\
\hline प्वाह & ष्वाह & - & $29=1 / 6$ & - & $100 / 6$ & عادى & \\
\hline $9 / 8=$ & $9 / 8=$ & - & $45=/ 74$ & - & - & 5C ميلى متر & ارتفاع بوته \\
\hline - & - & - & $15 / 20$ & - & $98 / 5$ & TIT ميلى متر & \\
\hline
\end{tabular}

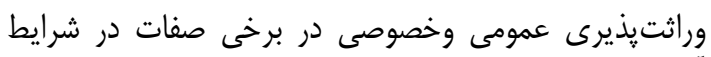

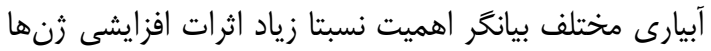



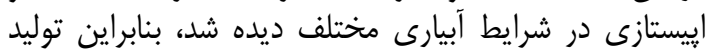

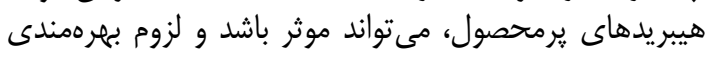


ير برنگ تر مى كند.

$$
\text { تشكر و قدردانى زبلى }
$$

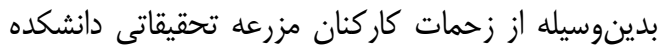

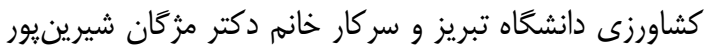

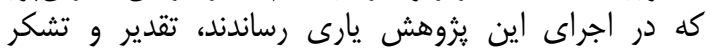
مى گردد.
اثر متقابل نسل× محيط براى تمامى صفات اندازمخيرى

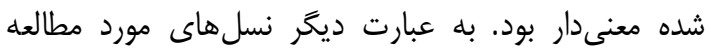

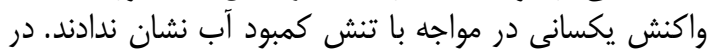

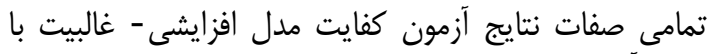

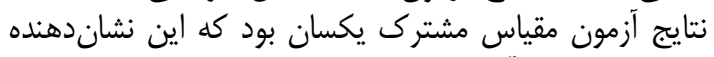

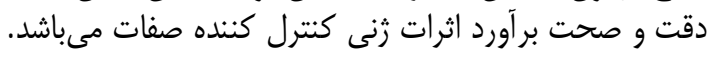

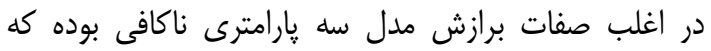

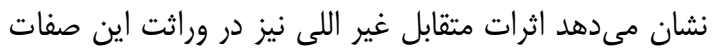

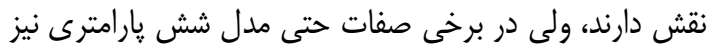

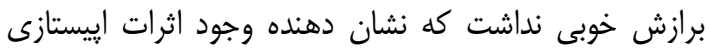

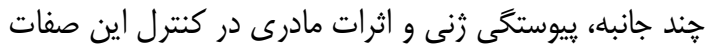

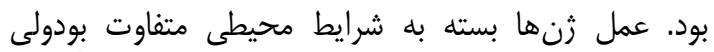

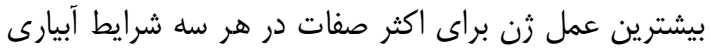

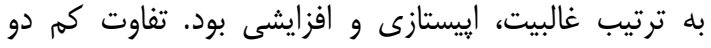



1. Ahmad, M., M. Saleem, M. Ahsan and A. Ahmad. 2016. Genetic analysis of water-deficit response traits in maize. Genetics and Molecular Research, 15(1): 1-10.

2. Al-Naggar, A.M.M, M.M.M. Atta, M.A. Ahmed, and A.S.M. Younis. 2016. Genetic variance, heritability and selection gain of maize (Zea mays L.) adaptive traits in high plant density combined with water stress. Journal of Applied Life Sciences International, 7(2): 1-17.

3. Allen, R.G., L.S. Pereira, D. Raes and M. Smith. 1998. Crop evapotranspiration: guidelines for computing crop water requirements. FAO. Irrigation and Drainage Paper. Rome.

4. Amer, E.A., A.A. El-Shenawy and H.E. Mosa. 2002. A comparison of four testers for the evaluation of maize yellow inbreds. Egyptian Journal of Applied Science, 17: 597-610.

5. Araus, J.L., M.D. Serret and G.O. Edmeades. 2012. Phenotyping maize for adaptation to drought. Frontiers in Physiology, 3: 1-20.

6. Blum, A. 1988. Plant Breeding for Stress Environment. CRP Press Incorporation, Florida, USA

7. Blum, A. 1988. Breeding crop varieties for stress environment. Critical Review in Plant Science, 2: 199-238.

8. Chaudhary, H.K., V. Kaila and S.A. Rather. 2014. Maize. Springer. New York.

9. Chohan, M.S.M. 2012. Genetic basis of drought tolerance and other plant traits in Zea mays L. PhD thesis, Department of Plant Breeding and Genetic, University of Agriculture, Faisalabad, Pakistan.

10. Comstock, R.E. and Robinson, H.F. 1948. The Components of genetic variance in populations of biparental progenies and their use in estimating the average degree of dominance. Biometrics, 4: 254266.

11. Dabholkar, A.R. 1992. Elements of Biometrical Genetics. Ashok Kumar Mittal Concept Publishing Company. New Dehli, India.

12. Ghahfarrokhi, A.R., N. Khodabandeh, A. Ahmadi and A. Bankehsaz. 2004. Study on effect of drought resistance in cereals. CAB International. pp: 27-52.

13. Gong, F., X. Wu, H. Zhang, Y. Chen and W. Wang. 2015. Making better maize plants for sustainable grain production in a changing climate. Frontiers in Plant Science, 6: 1-6.

14. Gonzalez, A., V. Bermejo and B.S. Gomeno. 2010. Effect of different physiological traits on grain yield in barley grown under irrigated and terminal water deficit conditions. Journal of Agriculture Science, 148: 319-328.

15.Griffing, B. 1956. Concept of general and specific combining ability in relation to diallel crossing system. Australian Journal of Biological Science, 9: 463-493.

16.Hallauer, A.R. and J.B. Miranda. 1988. Quantitative Genetics in Maize Breeding. 2nd ed. Iowa State University Press, US.

17.Hamidi, J. and Z. Khodarahmpour. 2011. Evaluation of drought Tolerance in different growth stage of maize (Zea mays L.) inbred lines tolerance indices. African Journal of Biotechnology, 10: 1348213490.18

18. Hayman, B.I. 1954. The theory and analysis of diallel crosses. Genetics, 39: 789-809.

19. Hayman, B.I. and K. Mather. 1955. The description of genetic interaction in continuous variation. Biometrics, 11: 69-82.

20. Heidari, M. 2006. Plant Response to Environmental Stresses. Aras Rayaneh Publication (In Persian).

21. Hopkins, W.G. and N.P. Huner. 2004. Introduction to Plant Physiology. $3^{\text {th }}$ ed. John Wiely and Sons Incorporation. New York.

22. Hugh, J.E. and R.F. Davis. 2003. Effect of drought stress on leaf and whole canopy radiation use efficiency and yield of maize. Agronomy Journal, 95: 688-696.

23. Iqbal, A.M., F.A. Nehvi, S.A. Wani, R. Qadir and Z.A. Dar. 2007. Combining ability analysis for yield and yield related traits in maize (Zea mays L.). International Journal of Plant Breeding and Genetic, 1: 101-105.

24. Jacob, H. and G. Clark. 2002. Methods of Soil Analysis. Journal of Soil Science, Madison, Wisconsin, USA. 1692 pp.

25. Kearsy, M.J. and H.S. Pooni. 1996. The Genetic Analysis of Quantitative Traits. Chapman and Hall, London, UK.

26. Khan, N.H., M. Ahsan, M. Naveed, H.A. Sadaqat and I. Javed. 2016. Genetics of drought tolerance at seedling and maturity stages in Zea mays L. Spanish Journal of Agricultural Research, 14(3): 1-11.

27. Khavari Khorasani, S., P. Dorri, M. Vali Zadeh and P. Taheri. 2014. Investigation the heritability and gene effects and some agronomic traits of maize (Zea mays L.). Plant Genetic Research, 1(2): 33-42 (In Persian).

28. Lgnaciuk, A. and D. Mason-Dcroz. 2014. Modeling Adaptation to Climate Change in Agriculture. OECD Food. Agriculture and Fisheries Paper. OECD Publishing.

29. Mather, K. and J.L. Jinks. 1982. Biometrical genetic. $3^{\text {th }}$ ed. Chapman and Hall, London, UK.

30. Mihailov, M.E. and A.A. Chernov. 2006. Using double haploid lines for quantitative trait analysis. Maize Genetics Cooperation Newsletter, 80: 16-30. 
31. Mirmohammady Maibody, S.A.M., P. Golkar and M. Golabadi. 2015. Plant Responses to Drought Stress. Sanati Esfahan Jahad Daneshgahi Publication (In Persian).

32. Muraya, M.M., C.M. Ndirangu and E.O. Omolo. 2006. Heterosis and combining ability in diallel crosses involving maize (Zea mays L.) S1 lines. Australian Journal of Experimental Agriculture, 46: 387-394.

33. Nelder, J.A. 1960. The estimation of variance components in certain types of experiment on quantitative genetics. Biometrical genetics, 139-158.

34. Ojo, G.O.S., D.K. Adedzwa and L.L. Bello. 2007. Combining ability estimates and Heterosis for grain yield and yield component in maize (Zea mays L.). Journal of Sustainable Development in Agriculture and Environment, 3: 49-57.

35. Rabbani, J. and Y. Emam. 2012. Yield response of maize hybrids to drought stress at different growth stage. Journal of Crop Production and Processing, 2(1): 65-78 (In Persian).

36. Ravikant, P.P. and P. Chandrakant. 2006. Gene effects for metric traits in quality protein maize (QPM) (Zea mays L.). Crop Improvement, 33: 94-101.

37. Ribaut, J.M., J. Betran, P. Monneveux and T. Setter. 2009. Drought tolerance in maize. Springer, New York.

38. Saeed, M.T., M.Saleem, and M. Afzal. 2000. Genetic analysis of yield and its components in maize diallel crosses (Zea mays L.). International Journal of Agriculture and Biology, 2(4): 376-378.

39. Schaap, M.G., F.J. Leij and M.T. Van Genuchten. 2001. ROSETTA: a computer program for estimating soil hydraulic parameters with hierarchical pedotransfer functions. Journal of Hydrology, 251: 163-176.

40. Shahrokhi, M., S. Khavari Khorasani and A. Ebrahimi. 2011. Generation mean analysis for yield and yield components in Maize (Zea mays L.). Journal of Plant Physiology and Breeding, 1(2): 59-72.

41. Shahrokhi, M., S. Khavari and A. Ebrahimi. 2013. Study of genetic component in various maize (Zea mays L.) traits, using generation mean analysis method. International Journal of Agronomy and Plant Production, IJAPP: 13-585.

42. Singh, P.K. and A.K. Roy. 2007. Diallel analysis of inbred lines in maize (Zea mays L.). International Journal of Agricultural Science, 3: 213-216.

43. Snedecor, G.W. and W.G. Cochran. 1989. Statistical methods, 8thEdn. Ames: Iowa State Univ. Press Iowa

44. Sprague, G.F. 1963. Statistical Genetics and Plant Breeding. National Academy of Science, National Research Council Publishing.

45. Srdic, J., Z. Pajhc and S.S. Mladenovic-Drinic. 2007. Inheritance of maize grain yield components. Maydica, 52(3): 261-264.

46. Taize, L. and E. Zeiger. 2006. Stress Physiology. In: Plant physiology. 4th ed. Sinauer Associates, 671-681.

47. Wattoo, F.M. 2013. Genetics of physio-agronomic traits iv maize under water deficit condition, PhD thesis, Departement of Plant Breeding and Genetic, University of Agriculture, Faisalabad, Pakistan.

48. Wu, G.H. 1987. Analysis of genetic effect for quantitative characters at different development states in maize. Genetics, 18: 69-69.

49. Yan, W., Y. Zhong and Z. Shangguan. 2016. Evaluation of physiological traits of summer maize under drought stress. Acta Agriculture Scandinavica, Section B Soil and Plant Science, 66: 133-140.

50. Yang, Z., S. Blankenagel, V. Avramova, C.C. Schön and E. Grill. 2018. Generation plants with Improved water use efficiency. In: Dreselhaus, T. and Hücklhoven, R. (eds.). Biotic and Abiotic Stress Responses in Crop Plants. MDPI, pp: 77-90. 


\title{
Genetic analysis of Agronomic Traits in Generations Derived from the Cross of MO17 and B73 Maize Inbred Lines Under Water Deficit Stress
}

\author{
Armin Vahed Rezaei ${ }^{1}$, Saeid Aharizad ${ }^{2}$, Majid Norouzi ${ }^{3}$ and Khosro Mafakheri ${ }^{4}$ \\ 1- Ph.D. Student of Genetic and Plant Breeding, University of Mohaghegh Ardabili \\ 2- Professor, Departement of Plant Breeding and Biotechnology, Faculty of Agriculture, University of Tabriz \\ (Corresponding author: s.aharizad@yahoo.com) \\ 3- Associate Professor, Departement of Plant Breeding and Biotechnology, Faculty of Agriculture, University of \\ Tabriz \\ 4- Ph.D. of Plant breeding (Molecular genetics and genetic engineering), Pars Holding Company, Tehran - \\ Received: May 5, $2020 \quad$ Accepted: November 12, 2020
}

\begin{abstract}
The selection of suitable yielding maize cultivars to water deficit is one of the main objectives of the breeding programs. Therefore, selection of a suitable breeding method for better productivity from genetic potential of stress tolerance and understanding the type of controlling genes action and their inheritance is very important. In this regard, genetic analysis of some of the agronomic traits of the generations resulting from cross between corn inbred lines MO17 (Tolerant to stress) and B73(stress sensitive) including: $\mathrm{F}_{1}, \mathrm{~F}_{2}, \mathrm{~F}_{3}, \mathrm{BC}_{1}$ and $\mathrm{BC}_{2}$ along with parents in three irrigation conditions (normal, 50 and 75 millimeter evaporation from class A evaporation pan) was evaluated in 2017-2018. Experiment was conducted in each irrigation conditions in a randomized complete block design with 20 replications. The traits including grain yield per plant, biomass per plant, 1000 grain weight, ear diameter, ear weight and rows number were measured. Combined analysis revealed that there was a significant difference between the studied generations for maize agronomic traits. The results of the scaling test indicated the existence of epistatic effects for most traits such as grain yield per plant, biomass per plant and 1000 grain weight under different irrigation conditions In most of the traits such as grain yield per plant, biomass per plant, row number per ear and 1000-grain weight, dominance effect was significant at 5\% and $1 \%$ probability level. About rows number three-parameter model was the best fit model, in all three conditions. For most of studied traits, six parameters models were significant in different irrigation conditions, but was not significant for plant height under normal irrigation conditions and $75 \mathrm{~mm}$ evaporation due to maternal effects, gene association and multiple chi-square epistasis. Estimation of the dominance degree above 1 for grain yield and less than 1 for ear diameter in all three irrigation conditions indicated that there was a phenomenon of over dominance and incomplete dominance in the control of them. High levels of genetic broad sence and narrow sence heritability suggested that genetic effects and additive effects have a greater role in the inheritance of 1000 grain weigth in all three irrigation conditions.
\end{abstract}

Keywords: Dominance, Gene effects, Generation mean analysis, Heritability, Maize 